\title{
Blast Injuries In Bangladesh
}

\section{Rahman $\mathbf{M H}^{1}$}

Blast injuries are very common now a days in Bangladesh for various socio political unrest.

Blast injuries are physically and psychologically devastating and dreadful. ${ }^{1}$ The explosions of bombs have caused serious unique patterns of injuries seldom seen in other etiological injuries. Most severely injured arrive after the less injured who go directly to the closest hospitals. Predominant injuries involve multiple penetrating injuries, blunt trauma and burns. Explosions occurring due to petrol bomb and cocktails in confined spaces like bus transport, CNG vehicles etc are associated with greater morbidity and mortality. All bomb events could have the potential for chemical and/or radiological contamination. Blast injuries are complex types of physical trauma ${ }^{2}$ caused by the detonation of explosives and are always a challenge to physicians and surgeons especially when it comes to rehabilitative care. Metal objects such as nails or steel balls are added to the charge in order to increase the fragmentation effect. In our country explosions in confined spaces and vehicles yielded more deaths and injuries. Analysis of blast incidents indicated that less injured patients often arrive at the hospital first, as they took themselves to the nearest hospital. The most severely injured arrived later. If there is a structural collapse, there will be more serious injuries that arrive more slowly. ${ }^{3}$

\section{Categories of Blast Injuries:}

Irrespective of the type of device used and the distance from the source, the effects of blasts cause injuries that fall into five categories ${ }^{3,4}$ - (1) Primary Blast Injuries (2) Secondary Blast Injuries (3) Tertiary Blast Injuries (4) Quaternary Blast Injuries (5) Quinary Blast Injuries.

Primary blast injuries: Primary blast injuries take place when the blast overpressure reaches the person and the transmitted forces exert their effect on the body, causing direct tissue damage. These are especially likely when a person is close to exploding munitions. The ears are most often affected by the overpressure, followed by the lungs and the hollow organs of the gastrointestinal tract, pulmonary damage and air embolization, hollow viscus injury. ${ }^{4}$

Secondary blast injuries: Most casualties are caused by secondary injuries. Secondary blast injuries are created by debris that is physically displaced by the blast overpressure or blast winds, from projectiles (bomb fragments, flying debris)- Penetrating trauma, fragmentation injuries, blunt trauma. These injuries are caused by fragmentation and other objects propelled by the explosion. ${ }^{1,4}$ These injuries may act upon any part of the body and sometimes result in penetrating trauma with visible bleeding. At times the propelled object may become embedded in the body, obstructing the loss of blood to the outside. However, there may be extensive blood loss within the body cavities.

Tertiary blast injuries: Tertiary blast injuries are caused when a person is physically displaced by the force of the peak overpressure and blast winds and sustains blunt trauma injury such as closed head injuries, blunt abdominal trauma, tissue contusions, or fractures. ${ }^{4,5}$ Additionally, collapse of surrounding structures confers a raised risk of severe tertiary blast injury (i.e., head trauma, traumatic asphyxia, and crush injuries). Commonly, small puncture wounds that represent secondary-blast injuries hide these fragments and severe underlying injuries. Young children, because they weigh less than adults, are at particular risk of tertiary injury.

1. Prof. Dr. M. Hasibur Rahman

Professor \& Head, Department of Dermatology \& VD, Community Based Medical College Bangladesh.

*Address of correspondence

Email : dr_cosmoderma@yahoo.com

Mobile: 01711318709 
Quaternary blast injuries: Quaternary blast injuriesoccasionally termed miscellaneous blast injuries-are caused directly by the explosion but are not classified as primary, secondary, or tertiary injury. They include but are not limited to burns, toxic substance exposures (e.g., radiation, carbon monoxide poisoning, cyanide poisoning), asphyxia, and psychological trauma. These include flash burns, crush injuries and respiratory injuries. In this injury traumatic amputations quickly result in death, and are thus rare in survivors, and are often accompanied by significant other injuries. The rate of eye injury may depend on the type of blast. ${ }^{4}$ Psychiatric injury, some of which may be caused by neurological damage incurred during the blast, is the most common quaternary injury, and post-traumatic stress disorder may affect people who are otherwise completely uninjured. ${ }^{4,5}$

Quinary blast injuries: Lastly is the quinary pattern of injury. In these injuries the patients manifest hyperpyrexia, diaphoresis, low central venous pressure, and a positive uid balance. ${ }^{6}$

\section{Initial Treatment and Management Strategies of Blast Injuries:}

The primary objective of initial management and treatment strategies are the restoration and stabilization of the patient's vital signs. ${ }^{4,7}$ Since terrorist attacks occur in civilian settings, the management and treatment of blast injuries are always a challenge. Irrespective of the extent of injury, initial treatment depends on the pattern of injury and should include surgical debridement, antibiotic therapy and, if necessary, tetanus prophylaxis in open injuries as well as the external stabilization of fractures with or without fasciotomy. Even when blast victims have small entrance wounds, surgeons should maintain a low threshold for performing thorough debridement to remove necrotic tissue and thoroughly irrigate as soon as possible. If possible, foreign bodies that are easily accessible or are accessible without the risk of further massive soft-tissue trauma should be removed. Obviously contaminated wounds should be irrigated with sterile saline and dressed with iodophore (Betadine) soaked sponges; once dressed, re-exposure should wait until operative exploration. ${ }^{5,7}$ Open wound treatment and secondlook operations at intervals of 48 to 72 hours are mandatory to obtain a clean and healthy wound environment. All open fractures are considered contaminated and should receive early antibiotic treatment (first generation cephalosporin and/or aminoglycocide, extended spectrum penicillin). ${ }^{7,8}$ With the emergence of an increasing number of multi-resistant organisms, wounds should be regularly swabbed and cultured in the post-primary period of treatment with a view to tailoring antibiotic provision on the basis of microbial sensitivity results. Extremity fractures should be splinted to provide mechanical stability and relieve pain.

Surgical Management: Initial debridement and bony stabilization ${ }^{5,8,10}$ should be done in the operating room to preserve life and limb. Following debridement, low-pressure pulsatile lavage may be employed to thoroughly irrigate the wound. ${ }^{4}$ Bony stabilization is often provided by external fixation with secondary conversion to definitive plate or intramedullary fixation. Following debridement and bony stabilization, soft tissue injury is generally addressed with creation of an antibiotic bead pouch or application of a vacuum wound dressing. Repeat debridement is planned every 24-72 hours, depending on the injury extent, until a stable soft tissue bed is attained Before and during each operative procedure, limb viability and feasibility of continued efforts to save the limb must be considered; the overall goal is to preserve potentially functional limbs jeopardizing the patient's overall health. ${ }^{4,10}$

\section{Conclusion:}

Blast injury (BI) presents unique diagnostic, and management challenges. ${ }^{9}$ Current patterns in extremist activity have increased the potential for casualties related to explosions. ${ }^{4,7}$ Very few health care providers in Bangladesh have experience treating patients with explosion-related injuries. Emergency care providers are therefore urged to learn more about the types of injuries that can result from blast injuries. Basic clinical information is 
needed for medical practitioners to know about the presentation, evaluation, management, and outcomes of Bls.

Treatment and management ${ }^{7,8,10}$ must be adapted to the extent of injury on a case-by-case basis in order to achieve a maximum reduction of mortality. Acute care providers (i.e., physicians, nurses, and ancillary staffs of emergency medicine, surgery, orthopaedics, and anaesthesiology), who are most likely to be the rst receivers of injured people, have an urgent responsibility to know and understand the diagnostic and management issues unique to blast injuries. Health-care systems must be able to provide care for the people and communities that are affected. ${ }^{8,10}$ Thus, every physician involved with emergency care needs to understand the unique injury patterns and management of explosion injuries.

\section{References}

1. Wolf, SJ, Babarata, VS., Bannett, CJ.m Pans, PT., and Cantrill, SV (2009): Blast Injuries. Lancet 374: 405-15.

2. Stephen J Wolf, Vikhyat S Bebarta, Carl J Bonnett, Peter T Pons, Stephen V Cantrill Blast injuries; Lancet 2009, 374: 405-15 Published Online July 23, 2009 DOI:10.1016/S01406736 (09)60257-9.

3. The American College of Emergency Physicians (ACEP) Bombings: Injury Patterns and Care Centers for Disease Control and Prevention (CDC). www.acep.org

4. Bieler, D., Hentsch, S., Franke, A. and Kollig,(2010) E. Current Strategies for the Treatment of Blast Injuries to the Extremities. MP-HFM-207-08.doc pp 1-16.

5. Blast Injuries fact sheet U.S. Department of Health and Human Services CDC's. Centers for Disease Control and Prevention of the blast fact sheets, http://emergency.cdc.gov/Blastinjuries

6. Kluger $Y$, Nimrod A, Biderman P, Mayo A, Sorkin $P$. The quinary pattern of blast injury. Am J Disaster Med 2007; 2: 21-25.

7. Centers for Disease control and Prevention (2006). Explosions and Blast Injuries. A Primer for clinicians: "http://www.bt.cdc.gov/masscasualties.asp"

8. Chaloner E (2005). "Blast injury in enclosed spaces: All doctors should know the basic management of patients injured by explosive blast". BMJ 331 (7509): 119-20. doi: $\quad 10.1136 / \mathrm{bmj} .331 .7509 .119$. PMC 558684. PMID 16009670

9. Stuhmiller, JH. (2010) Blast Injury Translating Research Into Operational Medicine. Edited by Santee WR, Fried KEI, US Army. Borden institute.

10. Treatment and Management: Blast Injuries emedicine. medscape.com/article/822587treatment www.bt.cdc.gov 